\title{
Anesthesiological considerations on a case of a giant sacrococcygeal teratoma with a hybrid surgical approach
}

\author{
S. Kramer, L. Garten, B. Opgen-Rhein, K. Rothe, C. Spies, R. Francis \\ Charité- Universitätsmedizin Berlin
}

Background: Sacrococcygeal teratomas (SCTs) can cause high output cardiac failure. Postnatal surgical interventions on SCTs have a much higher rate of survival than fetal interventions [Hedrick 2004]. Here we present a case of a giant SCT with a hybrid surgical approach including preoperative vascular embolization.
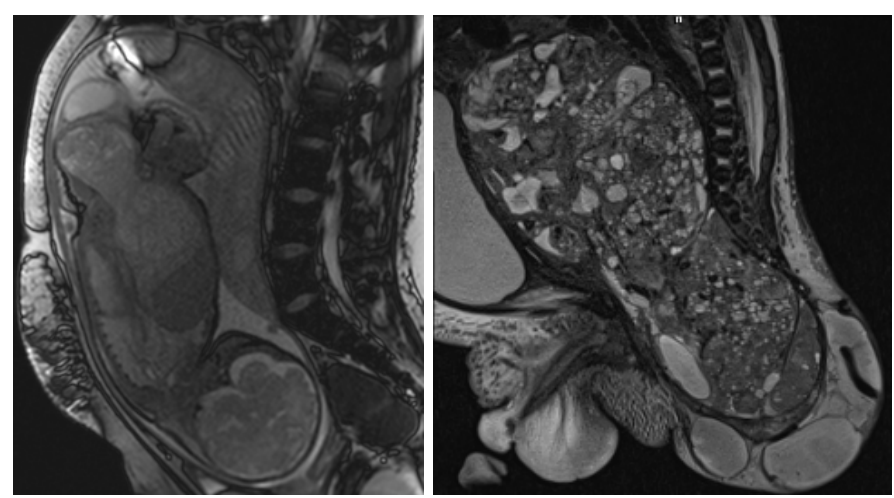

Picture 1, left: Maternal MRI with moderate SCT vascularization Picture 2, right: Postnatal MRI with detailed view of the SCR

Case report: Control obstetric sonogram revealed a giant SCT (Altman stage III) at 34 weeks gestational age with moderate tumor vascularization. In utero, fetal circulation was found normal. Postnatal angio-MRI revealed an extremely strong vascularization of the SCT. Vascularization of the SCT had changed dramatically with the end of placental perfusion and transition of to the postnatal circulation. Two arteries with the same size as the aorta originating directly from the left iliacal artery were embolized preoperatively. Therapeutic options, including palliative care, and risks of the procedure with long term impairments or fatal outcome, the parents' consent was obtained to perform hybrid surgical resection.

Intraoperatively, with continuous rate red cell and plasma transfusion, the infant was hemodynamically stable, during the early phase of surgical preparation of the tumor. However, during preparation of the large SCT vessels requiring manipulations on the embolized part of the tumor, the newborn's hemodynamic status deteriorated dramatically. Ubiquitous bleeding from all resected surfaces ensued rendering the resection of the tumor or even parts of the tumor impossible. Tranexamic acid was applied without any improvement of coagulation.
Extreme hyperkalemia and severe metabolic acidosis rapidly developed. In an attempt to correct hyperkalemia and acidosis, infusions of glucose/insulin, calcium chloride, hyperventilation, sodium bicarbonate and tromethamol were futile. Rapidly, the newborn demonstrated distinct signs of a systemic capillary leak syndrome including peritoneal swelling, lung edema and circulatory deterioration, requiring high dose catecholamine infusions. Pulmonary ventilation became literally impossible. At the point of repetitive epinephrine injections, a structured Team Time Out was performed and therapeutic aim was changed rigorously. The infant passed away in the arms of the parents.

\section{Discussion:}

This is the first report of a hybrid surgical approach to resect a giant SCT. The case demonstrates that prenatal assessment of SCT perfusion can differ significantly from the perfusion post partum, due to the overall transition to postnatal circulation of the newborn. Intraoperative hyperkalimia, acid-base dysbalance and significant capillary leak syndrome also suggests that this deterioration mimics ischemia reperfusion injury and redistribution of inflammatory cytokines and tumor degradation products into the systemic circulation that had been partially embolized preoperatively.

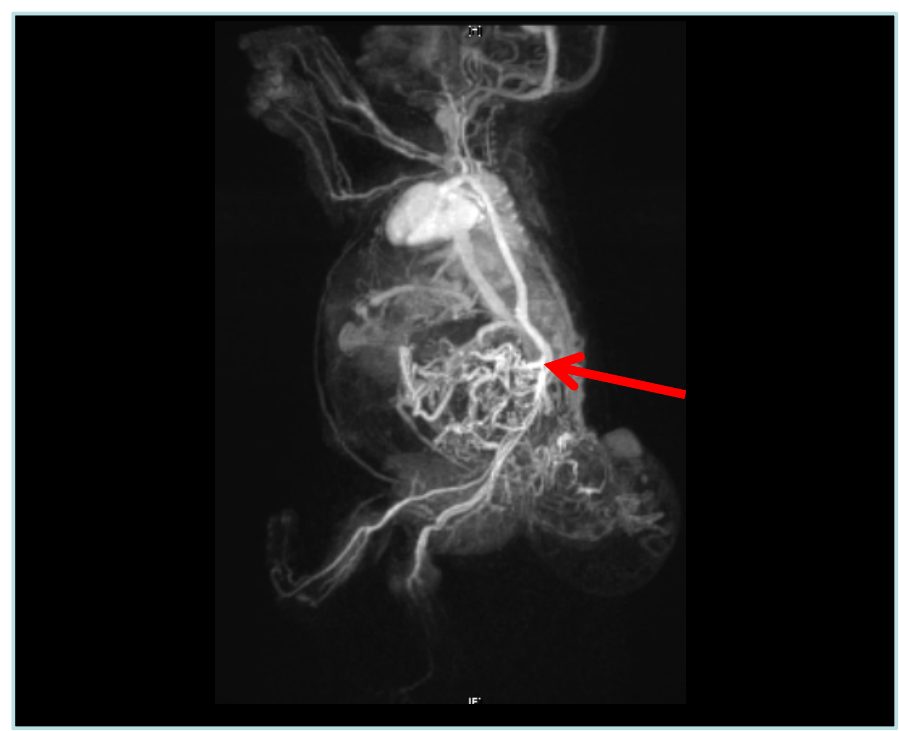

Picture 3:

Postnatal angio-MRI with extremely strong vascularization of the SCT.

Red arrow marking the embolized arteries

\section{Learning points:}

Prenatal and postnatal vascualarization of SCT can differ immensely due to conversion of fetal circulation

Hybrid procedure might be an option to facilitate surgical SCT resection

\section{References:}

Hedrick et al (2004) Sacrococcygeal Teratoma: Prenatal Assessment, Fetal Intervention,

and Outcome. J Ped Surgery 39: 430-438

Finnemore A, Groves A (2015) Physiology of the fetal and transitional circulation. Semin Fetal Neonatal Med 20:210-216 